\title{
Long term follow-up of drug resistant and drug susceptible tuberculosis contacts in a Low incidence setting
}

James Johnston ${ }^{1,2^{*}}$, Andrew Admon ${ }^{3}$, Amir Ibrahim² ${ }^{2}$ Kevin Elwood ${ }^{1}$, Patrick Tang ${ }^{4}$, Victoria Cook ${ }^{1,2}$ and Mark Fitzgerald ${ }^{2}$

\begin{abstract}
Background: Studies examining the transmission of multidrug-resistant tuberculosis (MDR-TB) strains have yielded conflicting results.

Methods: We examined transmission of MDR-TB strains using contact tracing data from a low incidence setting. Contacts of MDR-TB cases diagnosed in British Columbia, Canada, from 1990-2008 were identified through a provincial tuberculosis (TB) registry. Tuberculin skin test (TST) results and TB disease incident rates were determined for contacts. For comparison, TB disease incident rates and TST results were measured in close contacts of isoniazid mono-resistant (HMR-TB) and drug susceptible TB (DS-TB) cases.

Results: Of 89 identified close contacts of MDR-TB patients, 5 patients (6\%) developed TB disease and 42 (47\%) were TST positive. The incidence rate of TB disease (3\%, $p=0.31)$ and TST positivity $(49 \%, p=0.82)$ were similar in contacts of HMR-TB cases. Compared with MDR-TB contacts, DS-TB contacts had lower incidence rate of TB disease $(2 \%, p=0.04)$ and TST positivity $(32 \%, p<0.01)$. All MDR-TB contacts with culture positive TB diagnosed in follow-up were drug-susceptible; three of six HMR-TB contacts with culture positive TB were HMR-TB. Multivariate analysis demonstrated that contact with MDR-TB (adjusted OR 1.72; 95\%Cl 1.05-2.81) and HMR-TB (adjusted OR 1.99; 95\%Cl 1.48-2.67) was associated with TST positivity. In addition, adult age, male gender, BCG positivity, source case sputum smear positivity, foreign birth and fewer contacts per source case were significantly associated with TST positivity in the multivariate model.
\end{abstract}

Conclusion: Contacts of MDR-TB and HMR-TB patients in a low incidence setting show high rates of TST positivity and TB disease but low rates of drug resistance.

Keywords: Tuberculosis, Multidrug-resistant, Contact investigation, Epidemiology, Latent tuberculosis

\section{Background}

Multidrug-resistant tuberculosis (MDR-TB) refers to disease caused by Mycobacterium tuberculosis strains with in vitro resistance to both isoniazid (INH) and rifampin, two first line anti-tuberculosis drugs. The World Health Organization (WHO) estimates that there were 650,000 MDR-TB cases worldwide in 2008, corresponding to $3.6 \%$ of all tuberculosis (TB) cases [1]. MDR-TB is

\footnotetext{
* Correspondence: james.johnston@bccdc.ca

'Division of Tuberculosis Control, British Columbia Centre for Disease Control, Vancouver, BC, Canada

${ }^{2}$ Department of Medicine, University of British Columbia, Vancouver, BC, Canada

Full list of author information is available at the end of the article
}

associated with high rates of patient default, treatment failure and death [2]. Moreover, MDR-TB treatment is more costly when compared with first line therapy and requires enhanced clinical and laboratory support [1]. For these reasons, MDR-TB is a threat to achieving successful TB control [3].

Transmission of MDR-TB strains has been examined from several perspectives, including in vitro and animal studies, mathematical modeling and epidemiological investigation [4]. Results from epidemiological studies have been variable $[4,5]$. In studies utilizing molecular typing, drug-resistant strains demonstrate both increased and decreased clustering [6-12], while data from traditional

\section{Ciomed Central}


contact tracing studies note variable proportions of tuberculin skin test (TST) positivity and TB disease in MDR-TB contacts [13-20].

Contact tracing studies consistently report a significant proportion of contacts with TB disease that demonstrate a distinct resistance profile from their identified MDRTB source, indicating that not all supposed transmission events involve MDR-TB strains [12,14-20]. Despite this discrepancy, there is considerable evidence to support human-to-human MDR-TB strain transmission. Indeed over half of global MDR-TB cases are thought to result from primary transmission [21]. Yet appropriate preventative treatment of MDR-TB contacts remains unclear, as the relative infectivity MDR-TB strains is unknown and no high quality evidence exists to guide treatment of latent TB infection (LTBI) in contacts of drug resistant cases [22].

We examined local MDR-TB contact tracing outcomes in a low incidence setting. To better understand the transmissibility of MDR-TB strains, the proportion of close contacts of MDR-TB source that developed TB disease or were found to be TST positive in follow-up was compared to the proportion of close contacts of drug-susceptible TB (DS-TB) and isoniazid monoresistant TB (HMR-TB).

\section{Methods}

\section{Setting}

The study was conducted in British Columbia (BC), a Canadian province with a population of 4.4 million and a TB case rate of 7.1 per 100000 population per year [23]. The BC Centre for Disease Control (BCCDC) maintains a provincial population based TB registry that is informed of all TB cases through legal notification, as well as case notification through a centralized provincial mycobacteriology laboratory and pharmacy. The same agency dispenses all medications used to treat contacts. Contact tracing was performed according to Canadian guidelines and was recorded in the registry using standardized protocols [24].

\section{Bacterial culture and susceptibility testing}

M. tuberculosis was isolated from clinical specimens using the BacT/Alert mycobacterial culture detection system (BioMerieux, Durham, NC). Phenotypic susceptibility testing was performed using the BACTEC 460 radiometric method and interpreted as per Clinical and Laboratory Standards Institute (CLSI) recommendations $[25,26]$.

\section{Definitions}

For the purposes of this study, a source case was defined as the first household member to present with culturepositive pulmonary $\mathrm{TB}$ and drug susceptibility profile results. The source case date of diagnosis was defined as the date that the first culture positive sample was received by the laboratory. A close contact was defined as any individual identified as a "household contact" or "Type 1 contact" of a source case in the provincial TB registry. In this registry, both "household contact" and "Type 1 contact" represent a close contact and refer to household contacts or those sharing airspace with the source case for $>4$ hours per week. TSTs were performed according to Canadian guidelines [24]. A positive TST in a given contact was defined as any TST $\geq 5 \mathrm{~mm}$ measured $<3$ months before to $<1$ year after source diagnosis. Prior positive referred to a positive TST $\geq 3$ months before source diagnosis. A close contact identified by the source case without a TST measurement or TB disease diagnosis was recorded as no result. Contacts with a positive TST or with signs and symptoms of TB disease were screened with a chest $\mathrm{x}$-ray and in some cases, sputum examination. Contacts with TB disease was defined as a contact with $M$. tuberculosis complex demonstrated on culture or an individual with radiological, pathological or therapeutic responses consistent with TB disease as per established guidelines. Contacts with TB disease were not classified by their TST result.

\section{Data acquisition and analysis}

We accessed the BCCDC registry for all cases of MDRTB, HMR-TB and DS-TB diagnosed between 1990 and 2008. Close contacts of all MDR-TB cases were identified from the registry and their demographic profile, clinical features and TST results were recorded. For comparison, close contacts of DS-TB and HMR-TB were also identified with data recorded in a similar fashion. Only close contacts were recorded to limit bias introduced by enhanced contact tracing of drug-resistant sources. To further limit bias introduced by misclassification of close contacts, any sources with $>15$ close contacts were excluded from analysis.

The number of contacts with TB disease was recorded, and when available, contact resistance profiles were compared with their purported source case. TST positive or TST negative contacts with two sources listed within the same household in the same year were excluded from further TST analysis. BCG status was extracted for each contact, along with demographic variables. When BCG status was unavailable (in 36\% of cases), BCG status was estimated from www.bcgatlas.org using data on country of origin, age and year of entry to BC $[25,26]$.

\section{Statistical analysis}

Descriptive statistics were computed using Stata version 11.1 (StataCorp, College Station, Texas). The Wilcoxon Rank Sum test, chi-square test and Ficher's exact test 
were used in univariate analysis to assess statistical difference between variables (alpha 0.05). A multivariable logistic regression model was constructed to test the association between source resistance profile and TST positivity. Factors known to predict TST positivity, including adult age ( $\geq 18$ years), source smear status (positive), male gender, foreign birth, and BCG status were included in the model. After analysis of contact data, the number of contacts per source was added to the model, as the number of contacts varied significantly between HMR contacts and DS-TB contacts. A second model was constructed which excluded BCG status given the limited data for this variable. Odds ratios with 95\% confidence intervals were reported. Models were assessed with chi-square goodness-of-fit and ROC curve construction.

\section{Results}

\section{MDR-TB population}

We identified 35 MDR-TB cases from the BCCDC registry between 1990 and 2008 (Table 1). Contact tracing was not performed for 7 cases: 5 had extra-pulmonary TB, 1 case entered BC on MDR-TB therapy, and 1 case left $\mathrm{BC}$ before contact tracing was initiated. From the remaining 28 MDR-TB sources, 89 close contacts were identified, with a median of 3 total contacts per source (range 1-7) and median follow-up of 123 months (range
19-239). Of the 89 close contacts, 42 (47\%) were TST positive, $33(37 \%)$ were TST negative and $9(10 \%)$ were prior positive or no result (Table 2). Latent TB therapy was completed in 12 MDR-TB contacts, including 11 contacts that initiated preventative therapy tailored to the source case susceptibility profile. Five close contacts developed TB disease during follow-up; fully susceptible M. tuberculosis was isolated from 4 cases and the fifth case was diagnosed based on clinical criteria. All 5 contacts with TB disease were diagnosed within 3 months of source diagnosis and had not received preventative therapy.

\section{Isoniazid-resistant and drug-susceptible populations}

Between 1990 and 2008, contact tracing of 96 infectious HMR-TB source cases yielded 249 close contacts, of whom 121 (49\%) were TST positive (Table 2) and 8 (3\%) developed TB disease during follow-up. Of the six contacts with culture-confirmed TB, 3 developed HMR-TB, while 3 developed DS-TB (Table 3). DS-TB contact tracing over the same period yielded 7309 close contacts from 2895 sources (Table 2). There were 2321 contacts (32\%) with a positive TST and 168 (2\%) contacts with TB disease. Despite a common median of 3 close contacts per source case, the distribution of contacts varied significantly between DS-TB and HMR-TB $(\mathrm{p}<0.001)$.

Table 1 Characteristics of TST positive and negative contacts

\begin{tabular}{|c|c|c|c|c|c|}
\hline & \multirow{2}{*}{$\begin{array}{c}\text { DS-TB } \\
(\%)\end{array}$} & \multirow{2}{*}{$\begin{array}{c}\text { HMR-TB } \\
(\%)\end{array}$} & \multirow{2}{*}{$\begin{array}{c}\text { MDR-TB } \\
(\%)\end{array}$} & \multicolumn{2}{|c|}{ p-value vs DS-TB } \\
\hline & & & & HMR-TB & MDR-TB \\
\hline Source Cases & 2895 & 96 & 28 & & \\
\hline Household contacts & 7309 & 249 & 89 & & \\
\hline Median contacts/source & 3 & 3 & 3 & $<0.001 \wedge$ & $0.839 \wedge$ \\
\hline \multicolumn{6}{|l|}{ Source Characteristic } \\
\hline Source Smear Positive & $4709(64)$ & $139(56)$ & $57(64)$ & 0.005 & 0.939 \\
\hline \multicolumn{6}{|l|}{ Contact Characteristics } \\
\hline \multicolumn{6}{|l|}{ Demographics } \\
\hline Mean Age (Sd) & $32.9(21.2)$ & $28.9(20.4)$ & $26.7(19.1)$ & & \\
\hline Age Over 18 & $5489(75)$ & $170(68)$ & $57(64)$ & 0.013 & 0.015 \\
\hline Male Gender & $3521(48)$ & $112(45)$ & $43(48)$ & 0.32 & 0.98 \\
\hline \multicolumn{6}{|l|}{ Birth Country } \\
\hline Canadian born & $3163(43)$ & $87(35)$ & $60(67)$ & 0.001 & 0.001 \\
\hline Foreign born & $3609(49)$ & $154(62)$ & $23(26)$ & & \\
\hline Unknown & $536(7)$ & $8(3)$ & $6(7)$ & & \\
\hline \multicolumn{6}{|l|}{ BCG status* } \\
\hline Positive & $3340(46)$ & $132(53)$ & $44(49)$ & 0.101 & 0.752 \\
\hline Negative & $2977(41)$ & $94(38)$ & $42(47)$ & & \\
\hline Unknown & $991(14)$ & $23(9)$ & $3(3)$ & & \\
\hline
\end{tabular}

$\wedge$ Wilcoxon rank sum test.

* $22 \%$ of values were calculated using age, country of origin and information from www.bcgatlas.org. 
Table 2 Results of contact tracing

\begin{tabular}{lccccc}
\hline & $\begin{array}{c}\text { DS-TB } \\
(\%)\end{array}$ & $\begin{array}{c}\text { HMR-TB } \\
(\%)\end{array}$ & $\begin{array}{c}\text { MDR-TB } \\
(\%)\end{array}$ & \multicolumn{2}{c}{ p-value vs DS-TB } \\
\cline { 6 - 7 } & & HMR-TB & MDR-TB \\
\hline Source cases & 2895 & 96 & 28 & & \\
Close contacts & 7309 & 249 & 89 & & \\
TST positive & $2321(32)$ & $121(49)$ & $42(47)$ & $<0.001$ & 0.002 \\
Secondary case & $168(2)$ & $8(3)$ & $5(6)$ & 0.347 & 0.039 \\
Prior positive & $448(6)$ & $12(5)$ & $8(9)$ & 0.395 & 0.265 \\
No result & $475(6)$ & $9(4)$ & $1(1)$ & 0.067 & 0.045 \\
\hline
\end{tabular}

\section{Multivariate analysis of TST results}

All seven pre-specified variables predicted TST positivity in the logistic regression model, including adult age (OR 1.76; 95\% CI 1.51-2.06), male gender (OR 1.18; 95\% CI 1.05-1.32), BCG vaccination (OR 1.42; 95\% CI 1.231.64), foreign birth (OR 5.37; 95\% CI 4.55-6.33), source smear positivity (OR 1.23; 95\% 1.09-1.39), source HMRTB (OR 2.13; 95\% CI 1.57-2.90), and source MDR-TB (OR 1.75; 95\% CI 1.07-2.86) (Table 4). The number of

Table 3 Drug resistance patterns in source and secondary cases

\begin{tabular}{|c|c|c|c|c|}
\hline \multirow{2}{*}{$\begin{array}{l}\text { Related } \\
\text { pairs }\end{array}$} & \multicolumn{4}{|c|}{ Resistance pattern } \\
\hline & Isoniazid & Rifampin & Ethambutol & Streptomycin \\
\hline \multirow[t]{2}{*}{ MDR-TB1 } & 0.1 & 2.0 & 4.0 & S \\
\hline & $S$ & S & S & S \\
\hline \multirow[t]{2}{*}{ MDR-TB2 } & 0.1 & 2.0 & NR & $S$ \\
\hline & S & $S$ & S & $S$ \\
\hline \multirow[t]{2}{*}{ MDR-TB3 } & 0.1 & 2.0 & 4.0 & S \\
\hline & S & S & $S$ & S \\
\hline \multirow[t]{2}{*}{ MDR-TB4 } & 0.4 & 2.0 & 2.5 & $S$ \\
\hline & S & S & S & $S$ \\
\hline \multirow[t]{2}{*}{ MDR-TB5 } & 0.4 & 2.0 & 2.5 & $S$ \\
\hline & S & S & $S$ & S \\
\hline \multirow[t]{2}{*}{ HMR-TB1 } & 0.1 & S & S & S \\
\hline & 0.1 & S & $S$ & $S$ \\
\hline \multirow[t]{2}{*}{ HMR-TB2 } & 0.1 & S & S & S \\
\hline & 0.1 & $S$ & $S$ & S \\
\hline \multirow[t]{2}{*}{ HMR-TB3 } & 0.1 & $S$ & S & $S$ \\
\hline & $S$ & $S$ & $S$ & $S$ \\
\hline \multirow[t]{2}{*}{ HMR-TB4 } & 0.1 & $S$ & S & $S$ \\
\hline & S & S & $S$ & S \\
\hline \multirow[t]{2}{*}{ HMR-TB5 } & 0.1 & S & S & S \\
\hline & 0.1 & $S$ & $S$ & $S$ \\
\hline \multirow[t]{2}{*}{ HMR-TB6 } & 0.1 & $S$ & $S$ & $S$ \\
\hline & $S$ & $S$ & $S$ & $S$ \\
\hline
\end{tabular}

The first row in each pair represents source case sensitivity profile, while the second represents the secondary case susceptibility. $S=$ sensitive, $N R=$ no result. Resistance values reported in $\mathrm{microG} / \mathrm{microL}$.
Table 4 Multivariate analysis of factors associated with TST positivity

\begin{tabular}{lccc}
\hline Variable & Adjusted OR & p-value & $\mathbf{9 5 \% ~ C l}$ \\
\hline Age over 18 & 1.77 & $<0.001$ & $1.53-2.06$ \\
Male gender & 1.19 & 0.002 & $1.07-1.33$ \\
Foreign birth & 6.97 & $<0.001$ & $6.11-7.95$ \\
HMR-source & 1.99 & $<0.001$ & $1.48-2.67$ \\
MDR-source & 1.72 & 0.030 & $1.05-2.81$ \\
Smear positive source & 1.23 & 0.001 & $1.09-1.38$ \\
Increase in source contacts & 0.96 & $<0.001$ & $0.95-0.98$ \\
(per contact) & & & \\
\hline
\end{tabular}

close contacts attributed to the index case was included in the model because the distribution varied significantly between the HMR-TB and DS-TB groups, and because a lower threshold for classifying contacts as Type 1 may impact the rate of TST positivity. Each single increase in the number of close contacts was associated with a decrease in TST positivity (OR 0.96; 95\% CI 0.94-0.98). Estimates did not change significantly after BCG status was excluded from the model. Chi-square goodness of fit was non-significant for models that included and excluded BCG status, indicating that the goodness-of-fit for this model appeared adequate $(\mathrm{p}=0.32, \mathrm{p}=0.09$ respectively).

\section{Discussion}

Guidelines addressing latent TB infection (LTBI) treatment in MDR-TB contacts are vague and somewhat contradictory $[24,27,28]$. The WHO, citing the unknown efficacy of tailored preventative regimens, recommends observing contacts with careful clinical follow-up over two years [28]. The American Thoracic Society recommends observation or treatment with one of two regimens for 6-12 months [27]. These recommendations reflect the lack of high quality evidence required to direct decisions. Indeed, a recent Cochrane review failed to identify randomized control trials on MDR-TB contact tracing, while a 2006 systematic review found only two comparative studies suitable for analysis [23,29].

Given our results, combined with the discouraging completion rates of susceptibility-profile tailored regimens [30-32], IPT could be seen as a potential option in close contacts of MDR-TB patients in low incidence settings. This strategy, however, is not without risk [33]. MDR-TB transmission does occur in low incidence settings [12]. Moreover, in high incidence settings, the majority of patients with TB disease in MDR-TB contact populations develop MDR-TB $[16,18,19]$. Such contacts will not likely benefit from IPT. More importantly, however, this strategy could propagate drug-resistant disease by selectively killing drug sensitive organisms [34-37]. 
Thus, the risk of IPT appears to outweigh its benefit in MDR-TB contacts.

Our results demonstrate that MDR-TB contacts have higher rates of TST positivity compared with DS-TB contacts. This may reflect the increased transmissibility of MDR-TB strains. Alternatively, the high rates of TST positivity in MDR-TB and HMR-TB contacts may reflect differential distribution of unmeasured determinants for LTBI, such as source time-to-diagnosis, source cavitary disease or contact environmental and socioeconomic determinants. Indeed the development of DS-TB in all close contacts of MDR-TB with incident TB disease supports the notion that close contacts of drug-resistant source cases may be at higher risk for LTBI and TB disease independent of transmission from an identified MDR-TB source case.

There are several limitations in our study, the most significant being the lack of molecular typing data, which could help determine whether the discrepant drug susceptibility profiles were from strains with the same genotype. A second limitation is the small population of drug-resistant cases and contacts available for analysis. Previous studies examining traditional contact tracing in MDR-TB patients have demonstrated disease rates ranging from $0-8 \%$ in close contacts, consistent with our data [10-19]. However, in studies examining drugsusceptibility profiles, $62-92 \%$ of contacts that subsequently developed active disease had MDR-TB [13-16,18-20]. These rates are consistent with data demonstrating that $70 \%$ of secondary cases have the same genotype as their source case [38].

Finally, data on several determinants for TB infection are absent from analysis, including time-to-diagnosis, socioeconomic status and medical co-morbidities. Differential rates of TST positivity may be, in part, related to the differential distribution of these determinants. Nonetheless, our data suggests that risk for $M$. tuberculosis infection is higher in the contacts of drug resistant cases, and that thorough contact tracing should be performed in this population.

\section{Conclusions}

In conclusion, we described our experience with the contact tracing results of drug-resistant $\mathrm{TB}$ in a low prevalence region over nearly two decades. Our findings demonstrate that close contacts of MDR-TB at higher risk for LTBI and active TB. We suggest enhanced contact tracing in MDR-TB contacts, but caution against IPT in this population. Further research is urgently needed to determine the optimal management of LTBI in MDR-TB contacts.

\section{Abbreviations}

TB: Tuberculosis disease (also known as active tuberculosis); LTBI: Latent tuberculosis infection; MDR-TB: Multidrug resistant tuberculosis; DS-TB: Drug sensitive tuberculosis; HMR-TB: Isoniazid mono-resistant tuberculosis; INH: Isoniazid; IPT: Isoniazid preventative therapy; WHO: World Health Organization; TST: Tuberculin Skin Test.

\section{Competing interests}

The authors declare that they have no competing interests.

\section{Authors' contributions}

JJ: design, data acquisition, writing, statistical analysis. AA: statistical analysis, writing. Al: data acquisition. writing. PT: data acquisition, writing. VC: design, writing. KE: design, writing. MF: design, writing. All authors read and approved the final manuscript.

\section{Author details}

${ }^{1}$ Division of Tuberculosis Control, British Columbia Centre for Disease Control, Vancouver, BC, Canada. ${ }^{2}$ Department of Medicine, University of British Columbia, Vancouver, BC, Canada. ${ }^{3}$ Department of Internal Medicine, University of Michigan Health System, Ann Arbor, MI 48109, USA.

${ }^{4}$ Department of Pathology \& Laboratory Medicine, University of British Columbia, Vancouver, BC, Canada.

Received: 8 February 2012 Accepted: 18 October 2012

Published: 22 October 2012

\section{References}

1. World Health Organization. Multidrug and extensively drug-resistant TB (M/XDR-TB): 2010 Global report on surveillance and response. 2011. Available from: http://whqlibdoc.who.int/publications/2010/ 9789241599191_eng.pdf.

2. Johnston JC, Shahidi NC, Sadatsafavi M, Fitzgerald JM: Treatment outcomes of multidrug-resistant tuberculosis: a systematic review and meta-analysis. PLoS One 2009, 4:e6914.

3. Gandhi NR, Nunn P, Dheda K, Schaaf HS, Zignol M, van Soolingen D, et al: Multidrug-resistant and extensively drug-resistant tuberculosis: a threat to global control of tuberculosis. Lancet 2010, 375:1830-1843.

4. Borrell S, Gagneux S: Infectiousness, reproductive fitness and evolution of drug-resistant Mycobacterium tuberculosis. Int J Tuberc Lung Dis 2009, 13:1456-1466.

5. Dye C, Williams BG, Espinal MA, Raviglione MC: Erasing the world's slow stain: strategies to beat multidrug-resistant tuberculosis. Science 2002, 295:2042-2046.

6. Garcia-Garcia ML, Ponce De Leon A, Jimenez-Corona ME, Jimenez-Corona A, Palacios-Martinez M, Balandrano-Campos S, et al: Clinical consequences and transmissibility of drug-resistant tuberculosis in southern Mexico. Arch Intern Med 2000, 160:630-636.

7. Toungoussova OS, Sandven P, Mariandyshev AO, Nizovtseva NI, Bjune G, Caugant DA: Spread of drug-resistant Mycobacterium tuberculosis strains of the Beijing genotype in the Archangel Oblast, Russia. J Clin Microbiol 2002, 40:1930-1937.

8. Burgos M, DeRiemer K, Small PM, Hopewell PC, Daley CL: Effect of drug resistance on the generation of secondary cases of tuberculosis. $J$ Infect Dis 2003, 188:1878-1884

9. Fandinho FC, Kritski AL, Hofer C, Junior Conde H, Ferreira RM, Saad MH, et al: RFLP patterns and risk factors for recent tuberculosis transmission among hospitalized tuberculosis patients in Rio de Janeiro, Brazil. Trans $R$ Soc Trop Med Hyg 2000, 94:271-275.

10. Vazquez-Gallardo R, Anibarro L, Fernandez-Villar A, Diaz-Cabanela D, Cruz-Ferro E, del Molino ML $P$, et al: Multidrug-resistant tuberculosis in a low-incidence region shows a high rate of transmission. Int J Tuberc Lung Dis 2007, 11:429-435.

11. Godfrey-Faussett P, Sonnenberg P, Shearer SC, Bruce MC, Mee C, Morris L, et al: Tuberculosis control and molecular epidemiology in a South African gold-mining community. Lancet 2000, 356:1066-1071.

12. Nitta AT, Knowles LS, Kim J, Lehnkering EL, Borenstein LA, Davidson PT, et al: Limited transmission of multidrug-resistant tuberculosis despite a high proportion of infectious cases in Los Angeles County, California. Am J Respir Crit Care Med 2002, 165:812-817.

13. Attamna A, Chemtob D, Attamna S, Fraser A, Rorman E, Paul M, et al: Risk of tuberculosis in close contacts of patients with multidrug resistant tuberculosis: a nationwide cohort. Thorax 2009, 64:271. 
14. Teixeira $L$, Perkins MD, Johnson $J$, Keller R, Palaci M, Do Valle Dettoni $V$, et al: Infection and disease among household contacts of patients with multidrug-resistant tuberculosis. Int J Tuberc Lung Dis 2001, 5:321-328.

15. Kritski AL, Marques MJ, Rabahi MF, Vieira MA, Werneck-Barroso E, Carvalho CE, et al: Transmission of tuberculosis to close contacts of patients with multidrug-resistant tuberculosis. Am J Respir Crit Care Med 1996, 153:331-335.

16. Becerra MC, Appleton SC, Franke MF, Chalco K, Arteaga F, Bayona J, et al: Tuberculosis burden in households of patients with multidrug-resistant and extensively drug-resistant tuberculosis: a retrospective cohort study. Lancet 2011, 377:147-152.

17. Singla N, Singla R, Jain G, Habib L, Behera D, et al: Tuberculosis among household contacts of multidrug-resistant tuberculosis patients in Delhi, India. Int J Tuberc Lung Dis 2011, 15(10):1326-1330.

18. Grandjean L, Crossa A, Gilman RH, Herrera C, Bonilla C, Jave O, et al: Tuberculosis in household contacts of multidrug-resistant tuberculosis patients. Int J Tuberc Lung Dis 2011, 15(9):1164-1169.

19. Vella V, Racalbuto V, Guerra R, Marra C, Moll A, Mhlanga Z, et al: Household contact investigation of multidrug-resistant and extensively drug-resistant tuberculosis in a high HIV prevalence setting. Int I Tuberc Lung Dis 2011, 15(9):1170-1175.

20. Bayona J, Chavez-Pachas A, Palacios E, Llaro K, Sapag R, Becerra MC: Contact investigation as a means of detection and timely treatment of persons with infectious multidrug-resistant tuberculosis. Int J Tuberc Lung Dis 2003, 7:S501-S509.

21. World Health Organization: Antituberculosis drug resistance in the world: fourth global report. Geneva: World Health Organization; 2008.

22. BCCDC: Annual Report 2004-2008. Vancouver: BC Centre for Disease Control; 2008.

23. Fraser A, Paul M, Attamna A, Leibovici L: Treatment of latent tuberculosis in persons at risk for multidrug-resistant tuberculosis: systematic review. Int J Tuberc Lung Dis 2006, 10:19-23.

24. Long R, Ellis E: Canadian tuberculosis standards, Tuberculosis committee of the Canadian thoracic society of the Canadian lung association. 6th edition. 2010. Available from: www.phac-aspc.gc.ca/tbpc-latb/pubs/tbstand07-eng.php.

25. Clinical and Laboratory Standards Institute (CLSI): Susceptibility testing of mycobacteria, nocardiae, and other aerobic actinomycetes; approved standard. 2nd edition. Wayne, PA, USA: CLSI; 2011:M24-A2.

26. Zwerling A, Behr MAV, Brewer T, Menzies D, Pai M: BCG world atlas [internet]. Available from: www.bcgatlas.org.

27. Targeted tuberculin testing and treatment of latent tuberculosis infection. This official statement of the American Thoracic Society was adopted by the ATS Board of Directors, July 1999. This is a Joint Statement of the American Thoracic Society (ATS) and the Centers for Disease Control and Prevention (CDC): This statement was endorsed by the council of the infectious diseases society of America. (IDSA), September 1999, and the sections of this statement. Am J Respir Crit Care Med 2000, 161:\$221-S247.

28. World Health Organization: Guidelines for the programmatic management of drug-resistant tuberculosis. [Internet]. 2011. Available from: http://www.stoptb.org/assets/documents/resources/publications/technical/ tb_guidelines.pdf.

29. Fraser A, Paul M, Attamna A, Leibovici L: Drugs for preventing tuberculosis in people at risk of multiple-drug-resistant pulmonary tuberculosis. Cochrane Database of Systematic Reviews 2006, 19(2):CD005435.

30. Papastavros T, Dolovich LR, Holbrook A, Whitehead L, Loeb M: Adverse events associated with pyrazinamide and levofloxacin in the treatment of latent multidrug-resistant tuberculosis. CMAJ 2002, 167:131-136.

31. Horn DL, Hewlett D, Alfalla C, Peterson S, Opal SM: Limited tolerance of ofloxacin and pyrazinamide prophylaxis against tuberculosis. $N$ Engl $J$ Med 1994, 330:1241.

32. Younossian AB, Rochat T, Ketterer JP, Wacker J, Janssens JP: High hepatotoxicity of pyrazinamide and ethambutol for treatment of latent tuberculosis. Eur Respir J 2005, 26:462-464.

33. Cohen T, Lipsitch M, Walensky RP, Murray M: Beneficial and perverse effects of isoniazid preventive therapy for latent tuberculosis infection in HIV-tuberculosis coinfected populations. Proc Natl Acad Sci U S A 2006, 103:7042-7047.

34. Warren RM, Victor TC, Streicher EM, Richardson M, Beyers N, van Pittius NC $G$, et al: Patients with active tuberculosis often have different strains in the same sputum specimen. Am J Respir Crit Care Med 2004, 169:610-614
35. van Rie A, Victor TC, Richardson M, Johnson R, van der Spuy GD, Murray EJ et al: Reinfection and mixed infection cause changing Mycobacterium tuberculosis drug-resistance patterns. Am J Respir Crit Care Med 2005 172:636-642.

36. Colijn C, Cohen T, Murray M: Latent coinfection and the maintenance of strain diversity. Bull Math Biol 2009, 71:247-263.

37. Behr MA: Tuberculosis due to multiple strains: a concern for the patient? A concern for tuberculosis control? Am J Respir Crit Care Med 2004, 169:554-555.

38. Behr MA, Hopewell PC, Paz EA, Kawamura LM, Schecter GF, Small PM: Predictive value of contact investigation for identifying recent transmission of Mycobacterium tuberculosis. Am J Respir Crit Care Med $1998,158: 465-469$.

doi:10.1186/1471-2334-12-266

Cite this article as: Johnston et al.: Long term follow-up of drug resistant and drug susceptible tuberculosis contacts in a Low incidence setting. BMC Infectious Diseases 2012 12:266.

\section{Submit your next manuscript to BioMed Central and take full advantage of:}

- Convenient online submission

- Thorough peer review

- No space constraints or color figure charges

- Immediate publication on acceptance

- Inclusion in PubMed, CAS, Scopus and Google Scholar

- Research which is freely available for redistribution

Submit your manuscript at www.biomedcentral.com/submit
C Biomed Central 